\title{
Bifocal contact lenses: History, types, characteristics, and actual state and problems
}

\author{
Hiroshi Toshida \\ Kozo Takahashi \\ Kazushige Sado \\ Atsushi Kanai \\ Akira Murakami \\ Department of Ophthalmology, \\ Juntendo University School \\ of Medicine, Tokyo, Japan
}

\begin{abstract}
Since people who wear contact lenses (CL) often continue using CL even when they develop presbyopia, there are growing expectations for bifocal CL. To understand actual state and problems, history, types, and their characteristics are summarized in this review. Bifocal CL have a long history over 70 years. Recently, bifocal CL have achieved remarkable progress. However, there still is an impression that prescription of bifocal CL is not easy. It should also be remembered that bifocal CL have limits, including limited addition for near vision, as well as the effects of aging and eye diseases in the aged, such as dry eye, astigmatism, cataract, etc. Analysis of the long-term users of bifocal CL among our patients has revealed the disappearance of bifocal CL that achieved unsatisfactory vision and poor contrast compared with those provided by other types of CL. Changing the prescription up to 3 times for lenses of the same brand may be appropriate. Lenses that provide poor contrast sensitivity, suffer from glare, or give unsatisfactory vision have been weeded out. The repeated replacement of products due to the emergence of improved or new products will be guessed.
\end{abstract}

Keywords: bifocal contact lens, presbyopia, accommodation

\section{Introduction}

Prescription of contact lenses (CL) for persons with presbyopia is characterized by the following: 1) prescription of mildly corrective CL, 2) prescription of monovision $\mathrm{CL}, 3$ ) concomitant use of reading glasses and CL, 4) a shift to the use of glasses, and 5) use of bifocal CL. Since the 1990s, bifocal CL has made advances in design and optical performance, and products with a high prescription success rate have been released one after another. Bifocal CLs have achieved remarkable progress in the past decade. The increase of $\mathrm{CL}$ wearers with presbyopia and successive introduction of new products are some of the factors contributing to such progress. Although higher success rates for prescribing bifocal CL have been reported, there still is an impression that correct prescription of bifocal CL is not easy. The decrease in the number of $\mathrm{CL}$ wearers is similar to the change of accomodation with age. Here we summarize the history, the types and their characteristics, and the clinical results obtained with bifocal CL to demonstrate the actual state of use and the current problems. In addition, prospects for the future of bifocal CL will be discussed.

\section{History}

Bifocal CL have a long history. In 1938, Feinbloom from New York reported a segmented bifocal CL and a trifocal CL (Moss 1962). However, these lenses did not come into clinical use because measures for preventing their rotation were not taken. In 1957, DeCarle in London developed simultaneous-vision bifocal CL that were free from the problem of rotation, which became the basis for current bifocal CL (DeCarle 1989). In the latter half of the 1980s to the 1990s, nonspherical progressive multifocal CL (Stein 1990) and diffraction CL (Freeman and Stone 1987) were developed, and a large number of bifo- 

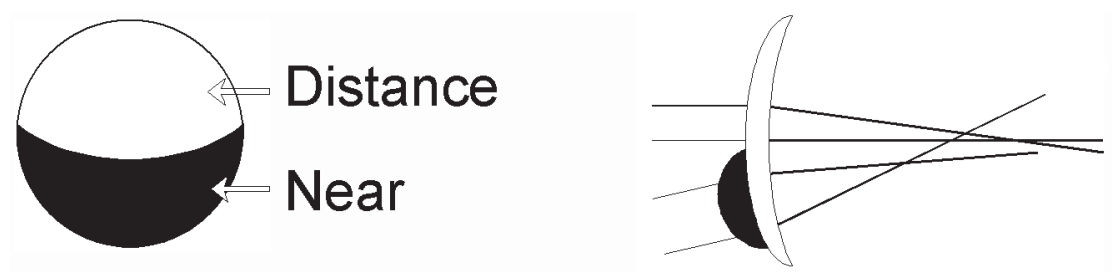

Figure I Diagram of an alternating vision RGP lens. The upper and lower parts of the lens provide distant and near vision, respectively.

cal products were released. Some of these CL subsequently showed a decrease or increase in the number of prescriptions, while others are no longer available because the manufacturers stopped producing them. For example, analysis of the long-term users of bifocal CL among our patients, as described below, has revealed the disappearance of old bifocal CL that achieved unsatisfactory vision and poor contrast compared with those provided by other types of CL.

In recent years, bifocal CL have undergone further improvement, including the development of modified monovision lenses with a difference in power between the right and left sides (Gohrmlet 1989) and bifocal soft contact lenses (SCL) in which the optical axis coincides with the line-ofsight (Tanaka et al 1995, 1996).

\section{Types of bifocal CL}

Bifocal CL can be classified into a) alternating vision and $b$ ) simultaneous vision types. With the former type, as with bifocal glasses, the wearer sees an object through either the distant or near vision part by moving the visual axis upward or downward, while both distant and near images are formed on the retina simultaneously with the latter type. Simultaneous vision bifocal $\mathrm{CL}$ are classified into refractive and diffractive types.

\section{Alternating or translating vision}

Simultaneous vision bifocal CL are also called segmented lenses (Figure 1). Like the lenses of bifocal glasses, distant and near vision are supported by the upper and lower parts of the lens, respectively. Therefore, it is necessary to move the visual axis downward for near vision, and image jumping tends to occur when crossing the boundary between the two parts of the lens. Centering is important, and to prevent rotation, a ballast prism is attached to the lower part of the lens or the truncation method is used.

\section{Simultaneous vision}

\section{Refractive lenses}

\section{Concentric type}

In the DeCarle type of lens (Figure 2), the central and peripheral parts of the lens are used for distant and near vision, respectively, while their functions are reversed in the Alges type of lens. In progressive multifocal CL, the power changes successively from the center toward the periphery. Light from both distant and near objects forms images on the retina simultaneously. The wearer sees both images and the brain decides which image should be selected. This is just like the way we differentiate the sounds we want to hear from background noise. This type of lens is free from the problem of rotation. However, since vision is influenced by the tilt or deviation of the lens, centering and appropriate fitting are important. In addition, there is a 5-zone bifocal lens with the central zone providing distant vision, which is surrounded by alternating near and distant vision zones.

\section{Nonspherical type}

The posterior surface (or the anterior and posterior surfaces) of the lens is nonspherical, and its central and paracentral parts provide distant and near vision, respectively, with lens power changing successively between these two parts (Figure 3). In the case of rigid gas permeable (RGP) CL, the lens moves on the cornea, and therefore the effect of alternating vision can also be obtained with infraversion.

$\mathrm{CL}$ in which the optical axis coincides

with the line of sight

In this design, the optical center is not at the center of the lens, but is shifted slightly in the nasal direction so that it coincides with the pupil (Figure 4). Since the visual axis passes through the near vision part of the lens, convergence and miosis occur at the time of near vision, so this type of lens produces good near vision.

\section{Diffractive lenses}

There is a concentric groove on the posterior surface of these lenses, which causes diffraction of some of the incident light rays (Figure 5). The image of a distant object is formed by refraction at the center of the lens, while that of a near object is formed as a primary diffractive image in the circular diffractive zone on the inner surface of the lens. 

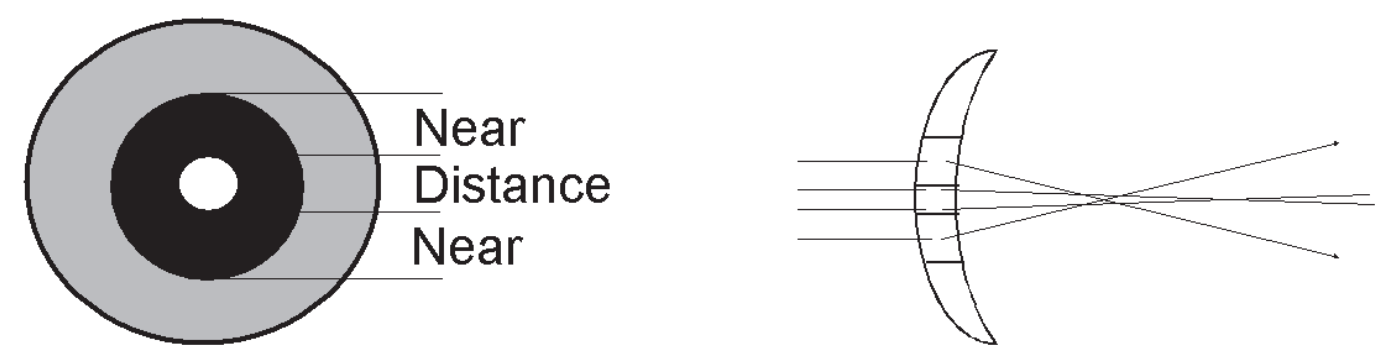

Figure 2 Diagram of a concentric SCL. In this design, distant and near vision are provided by concentric areas of the CL, which are classified as bifocal and progressive multifocal.
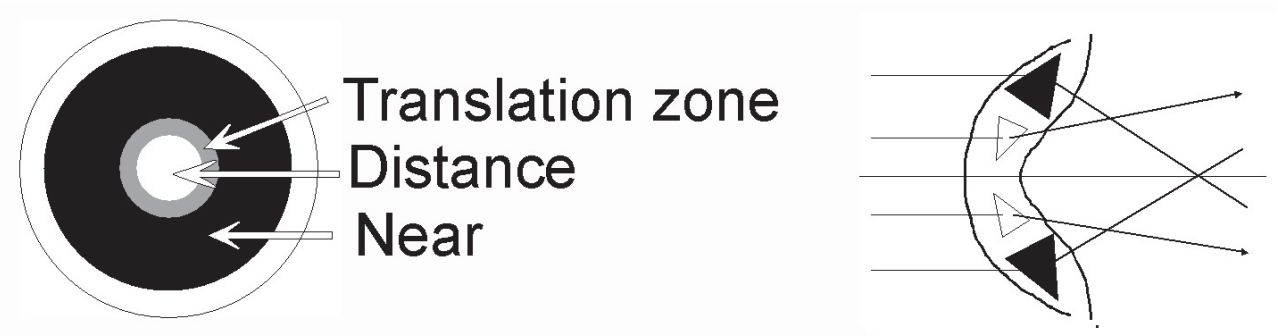

Figure 3 Diagram of a nonspherical lens. The posterior surface (or the anterior and posterior surfaces) of the lens is nonspherical, and the central and paracentral parts of the lens provide distant and near vision, respectively. The power changes successively between the two parts.
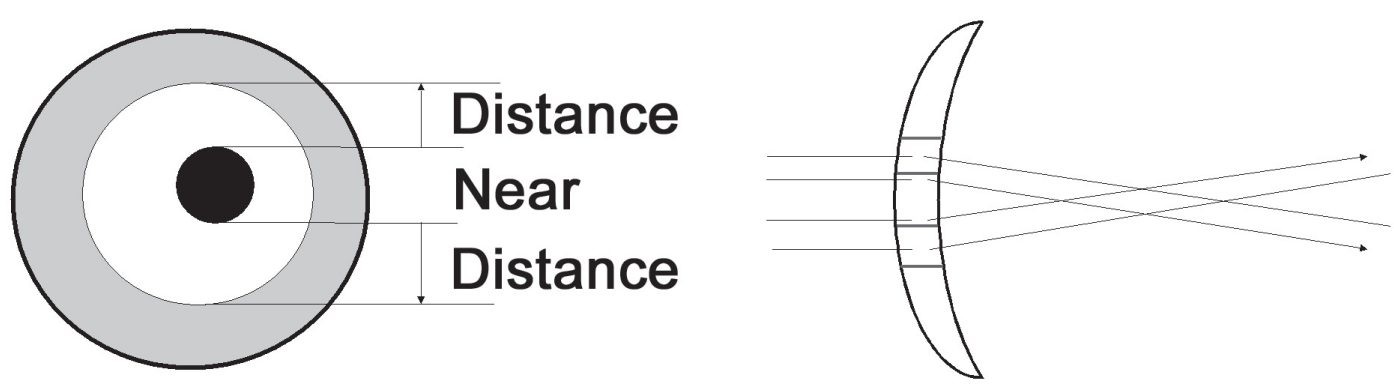

Figure 4 Diagram of a lens in which the optical axis coincides with the line of sight. In this design, the optical center of the lens is shifted slightly toward the nose so that it coincides with the line of sight. Since convergence and miosis occur at the time of near vision, this design is most suitable for near vision.
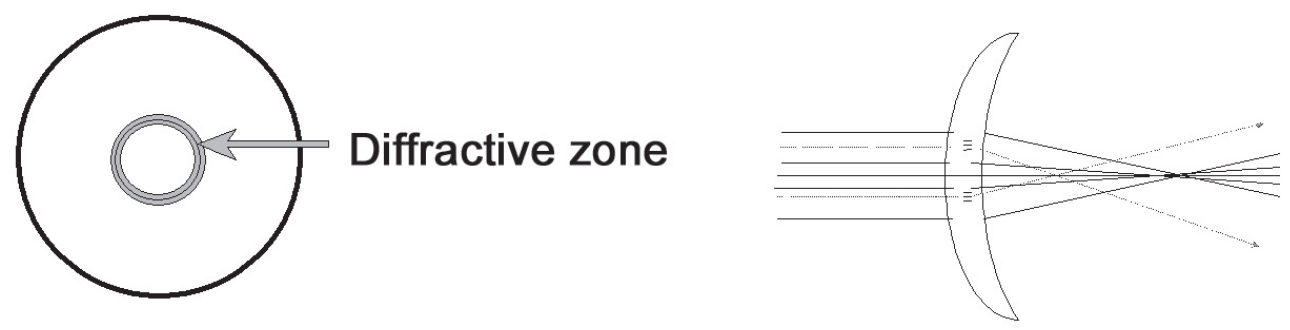

Figure 5 Diffractive SCL.There is a refractive part at the center, which is surrounded by many circular diffractive zones.A distant image is formed by refraction at the center, while a near image is formed in the diffractive zone on the inner surface of the lens. 
However, the contrast of diffractive images is poor and glare is often a problem.

\section{General characteristics of bifocal CL}

Prescription of bifocal CL is generally considered to be difficult and the success rate is low. This is presumably because various designs and types of bifocal CL are available: there is limited addition for near vision; contrast is poor, and it is difficult to see in dark places; glare may be problematic; vision is inferior to that provided by monofocal CL; the lenses are more expensive; and special techniques are needed for proper prescription. As has been indicated by a large number of authors, bifocal CL should only be prescribed by consideration of the history of CL use and after confirming by sufficient questioning why the wearer wants bifocal CL and whether distant or near vision is more important. Since both the prescription and type of lens often have to be changed, several kinds of lenses will need to be tried and patience is required to achieve success.

It was reported that the success rate of prescribing $\mathrm{CL}$ was $67 \%-83 \%$ after 3 months of follow up, which is not so low (Back et al 1989; Tanaka et al 1996; Takahashi and Sado 2002; Hamano 2002). However, it has also been reported more recently that the actual success rate for long-term wearers is lower, being around $30 \%-40 \%$ in most cases (Harris et al 2005; Hamano et al 2006).

\section{Long-term use of bifocal CL by our patients}

Data on our patients who had been wearing bifocal CL for 3 years or longer were analyzed to clarify the characteristics of bifocal CL, which previously were associated with a low success rate.

\section{Patients}

Among the 206 patients (409 eyes) for whom bifocal CL were prescribed during the 10 years between October 1997 and September 2006, 70 patents (137 eyes) continued to use the lenses for at least 3 years and were enrolled as the subjects of the present study. They accounted for $33.5 \%$ of all patients, and the percentage of males and females was $15 \%$ and $85 \%$, respectively. Their mean age at the time of lens prescription was $51.4 \pm 6.0$ years (mean \pm SD). Apart from a 16-year-old aphakic patient ( 1 eye) after cataract surgery, these patients were aged $44-65$ years, with a peak in the early fifties (Figure 6).

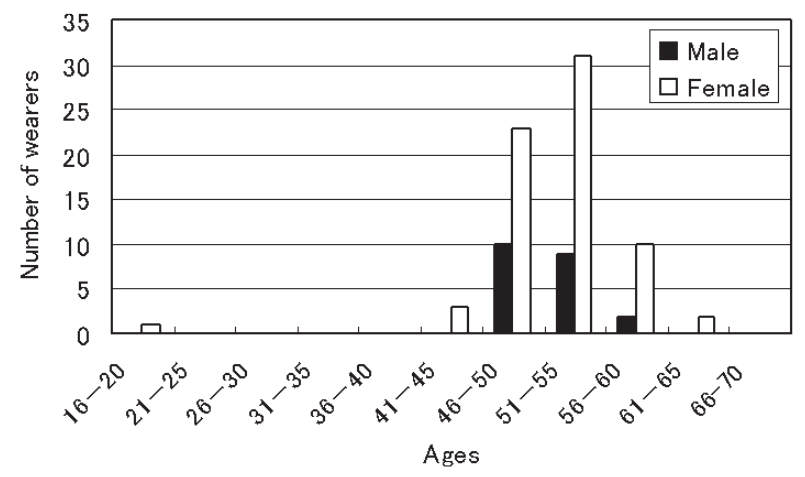

Figure 6 Number of bifocal CL wearers in each age.

\section{Types of bifocal CL}

The relationship between the type of monofocal lens used before the prescription of bifocal CL and the type of bifocal lens that could be worn was investigated (Table 1). As a result, it was confirmed that bifocal RGP CL was prescribed for all monofocal RGP lens wearers, while bifocal SCL was prescribed for monofocal SCL wearers, apart from one patient (2 eyes) who subsequently switched to bifocal RGP CL. Bifocal SCL was also prescribed for all of the patients with no prior history of CL use.

The relative spherical power of the bifocal $C L$ was in the order of high myopia (over $-6 \mathrm{D}, \mathrm{n}=50$ ) $>$ moderate myopia (over $-3 \mathrm{D}$ to $-6 \mathrm{D}, \mathrm{n}=44)>$ emmetropia to hyperopia $(n=31)>$ mild myopia $(-3 \mathrm{D}$ or less, $\mathrm{n}=11)$. Another one was aphakia.

\section{Results}

Visual acuity was measured with a Snellen chart. After 3 months of follow-up, the mean distant vision was 0.91 , the mean near vision was 0.78 , the mean addition for near vision was $+2.61 \mathrm{D}$, and the successful prescription rate was $83.0 \%$ (Table 2 ). At the time of final examination, the mean distant vision was $1.00 \pm 0.08$, the mean near vision was $0.69 \pm 0.07$, the mean addition for near vision was $+2.73 \pm 0.81 \mathrm{D}$, and the success rate was $33.5 \%$. The lens

Table I Selection of bifocal CLs at the initial prescription

\begin{tabular}{ll}
\hline Experience with CLs & Type of bifocal CLs \\
\hline RGP & RGP bifocal \\
103 eyes & 103 eyes \\
SCL & SCL bifocal \\
8 eyes & 8 eyes \\
No experience of CL & SCL bifocal \\
26 eyes & 26 eyes* \\
\hline
\end{tabular}

Notes: *:One subject (2 eyes) have changed to RGP bifocal CL later. Abbreviations: $\mathrm{CL}$, contact lenses; RGP, rigid gas permeable; $\mathrm{SCL}$, soft co ntact lenses. 
Table 2 Results of long-term use of bifocal CL in our department

\begin{tabular}{lll}
\hline & $\mathbf{3}$ months & $\mathbf{3}$ years \\
\hline Mean age (years-old) & $51.7 \pm 8.5$ & $51.4 \pm 6.0$ \\
Distant vision (Snellen acuity) & $0.93 \pm 0.07$ & $1.00 \pm 0.08$ \\
Near vision (Snellen acuity) & $0.78 \pm 0.15$ & $0.69 \pm 0.07$ \\
Addition for near vision (D) & $2.61 \pm 0.57$ & $2.73 \pm 0.81$ \\
Success rate & $83.0 \%$ & $33.5 \%$ \\
Change of prescription (times) & $1.5 \pm 0.5$ & $2.4 \pm 1.9$ \\
\hline
\end{tabular}

prescription was changed from $0-7$ times, with the mean frequency being $2.4 \pm 1.9$ times. The three major reasons for changing the prescription were unsatisfactory near vision, unsatisfactory distant vision, and blurred vision, so all these were related to the quality of vision. The five typical lens types that were initially prescribed frequently are displayed in Table 3. ForView ${ }^{\circledR}$ (Rainbow, Tokyo, Japan) and $\operatorname{Expert}^{\circledR}$ (Seed, Tokyo, Japan) were inferior to the other lenses with respect to near vision, and the mean frequency of prescription changes exceeded 3 for these lenses. As reported previously, contrast sensitivity examined using the Multivision Contrast Tester (MCT8000, Vistec, Weilburg, Germany) provided by bifocal CL tends to be poor in the middle to high spatial frequencies (Takahashi and Sado 2002). However, the contrast obtained with these two types of CL was even lower than with the other types (Figure 7). The number of patients wearing these lenses remarkably decreased to less than $15 \%$ around 3 years after, and the manufacturers subsequently discontinued both lenses. Conversely, other lenses had the advantages that both near and distant vision was satisfactory and the frequency of prescription changes was low.

\section{Discussion}

Since accommodation of the eye decreases with age, presbyopia is a problem we cannot avoid. When CL are prescribed, importance is attached to distant vision in younger wearers. However, it will become difficult for them to maintain good near vision when they become old enough to develop presbyopia. Since CL

Table 3A Major bifocal RGP lenses that were initially prescribed. Details of the CLs, number of the eyes, near and distant vision, the addition for near vision (D) and frequency of prescription changes. Visual acuity was measured with a Snellen chart. To analyze the data, each value was transformed to a logMAR visual acuity value. Data are re-calculated to Snellen visual acuity, again.

\begin{tabular}{|c|c|c|c|c|}
\hline Brand & Material & Design & At time of observation & No. of eyes \\
\hline More & RGP & Alternating & 3 Months & 6 \\
\hline (Rainbow Optical,Tokyo, Japan) & & (Segment) & 3 Years & $6(100.0 \%)$ \\
\hline Clair & RGP & Simultaneous & 3 Months & 134 \\
\hline (Rainbow Optical,Tokyo, Japan) & & (Nonspherical) & 3 Years & $46(34.3 \%)$ \\
\hline Expert & RGP & Simultaneous & 3 Months & 70 \\
\hline (Seed,Tokyo, Japan) & & (Nonspherical) & 3 Years & $10(14.3 \%)$ \\
\hline ForView & $\mathrm{SCL}$ & Simultaneous & 3 Months & 26 \\
\hline (Rainbow Optical, Tokyo, Japan) & & (Concentric) & 3 Years & $3(11.5 \%)$ \\
\hline Menifocal soft & $\mathrm{SCL}$ & Simultaneous & 3 Months & 24 \\
\hline (Menicon, Nagoya, Japan) & & (Optical axis coincides) & 3 Years & II (45.8\%) \\
\hline
\end{tabular}

Table 3B Major bifocal SCLs that were initially prescribed. Details of the CLs, number of the eyes, near and distant vision, the addition for near vision (D) and frequency of prescription changes. Visual acuity was measured with a Snellen chart. To analyze the data, each value was transformed to a logMAR visual acuity value. Data are re-calculated to Snellen visual acuity, again.

\begin{tabular}{|c|c|c|c|c|c|}
\hline Brand & Time of observation & Distant vision & Near vision & $\begin{array}{l}\text { Addition for near } \\
\text { vision (D) }\end{array}$ & $\begin{array}{l}\text { Prescription } \\
\text { changes }\end{array}$ \\
\hline \multirow[t]{2}{*}{ More } & 3 Months & $0.97 \pm 0.57$ & $0.79 \pm 0.12$ & $2.58 \pm 0.43$ & $0.9 \pm 0.8$ \\
\hline & 3 Years & $1.05 \pm 0.91$ & $0.91 \pm 0.71$ & $2.63 \pm 0.43$ & $2.6 \pm 2.6$ \\
\hline \multirow[t]{2}{*}{ Clair } & 3 Months & $0.86 \pm 0.63$ & $0.67 \pm 0.15$ & $2.00 \pm 0.00$ & $0.9 \pm 0.3$ \\
\hline & 3 Years & $0.97 \pm 0.81$ & $0.66 \pm 0.75$ & $3.43 \pm 0.47$ & $1.9 \pm 1.7$ \\
\hline \multirow[t]{2}{*}{ Expert } & 3 Months & $0.94 \pm 0.57$ & $0.64 \pm 0.24$ & $3.71 \pm 0.67$ & $1.7 \pm 0.8$ \\
\hline & 3 Years & $1.04 \pm 0.88$ & $0.62 \pm 0.74$ & $3.15 \pm 1.86$ & $3.2 \pm 1.9$ \\
\hline \multirow[t]{2}{*}{ ForView } & 3 Months & $0.80 \pm 0.36$ & $0.55 \pm 0.23$ & $2.38 \pm 0.36$ & $1.8 \pm 0.6$ \\
\hline & 3 Years & $0.82 \pm 0.84$ & $0.31 \pm 0.63$ & $2.46 \pm 0.46$ & $3.9 \pm 1.9$ \\
\hline \multirow[t]{2}{*}{ Menifocal soft } & 3 Months & $0.97 \pm 0.20$ & $0.98 \pm 0.08$ & $2.32 \pm 0.22$ & $0.6 \pm 0.1$ \\
\hline & 3 Years & $1.02 \pm 0.89$ & $0.79 \pm 0.08$ & $2.25 \pm 0.27$ & $2.6 \pm 2.1$ \\
\hline
\end{tabular}

Abbreviations: RGP, rigid gas permeable; SCL, soft contact lenses. 

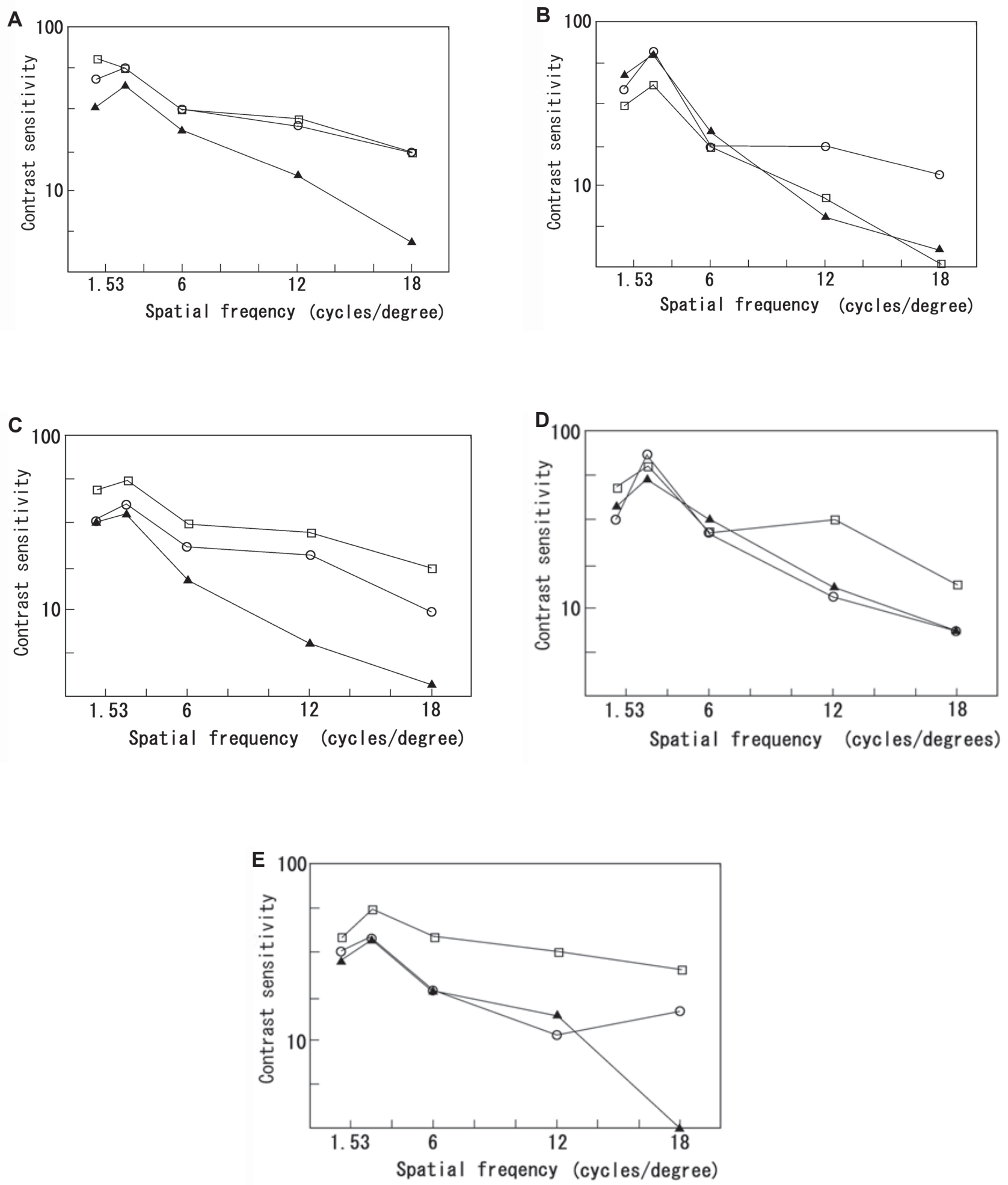

Figure 7 Contrast obtained with various bifocal CL. Results obtained by measurement of contrast while wearing bifocal CL using a multivision contrast tester (MCT8000, Vistec $\operatorname{lnc}$ ). Distant vision during the daytime $(\bigcirc)$, near vision during the daytime $(\bullet)$, and near vision at night $(\boldsymbol{\Delta})$. Data obtained at the time of wearing glasses were used as control values. A Eye glasses (control); B Simultaneous, Nonspherical type (RGP): Expert ${ }^{\circledR}$; C Simultaneous, Nonspherical type (RGP): Clair ${ }^{\circledR}$; D CL in which the optical axis coincides with the line of sight (SCL): E Menifocal soft ${ }^{\circledR}$; Simultaneous, Concentric type (SCL): ForView ${ }^{\circledR}$. 
has been widely used for more than 50 years, the percentage of CL wearers with presbyopia has been increasing year by year (Van Meter et al 2001). Under the present circumstances, however, many of CL wearers still stop using their lenses after the onset of presbyopia (Figure 8A) (Succeed 2004). The decrease in the number of CL wearers is similar to the change of accommodation with age (Figure 8B) (Donders 1864; Duane 1908; Clarke 1918; Ishihara 1919; Fukuda et al 1962). On the other hand, people with mild to moderate hyperopia who did not need glasses until the development of presbyopia generally tend to avoid reading glasses, so there are growing expectations for the use of bifocal CL among such people.

As accommodation decreases during long-term use of $\mathrm{CL}$, near vision also decreases, and becomes necessary to switch the patient to bifocal CL. Although prescription changes are essential for bifocal CL, it is recommended to alter the lens type if the frequency of such changes is too high. Because prescribing these lenses is complicated, there may be a tendency for bifocal CL not to be promoted actively (Harris et al 2005). Changing the prescription up to 3 times for lenses of the same brand may be appropriate. Lenses that provide poor contrast sensitivity, suffer from glare, or give unsatisfactory vision have been weeded out. We have experienced the repeated replacement of products due to the emergence of improved or new products.

Thanks to such progress, it was reported that more than half of the wearers used to monovision lenses selected bifo-

A
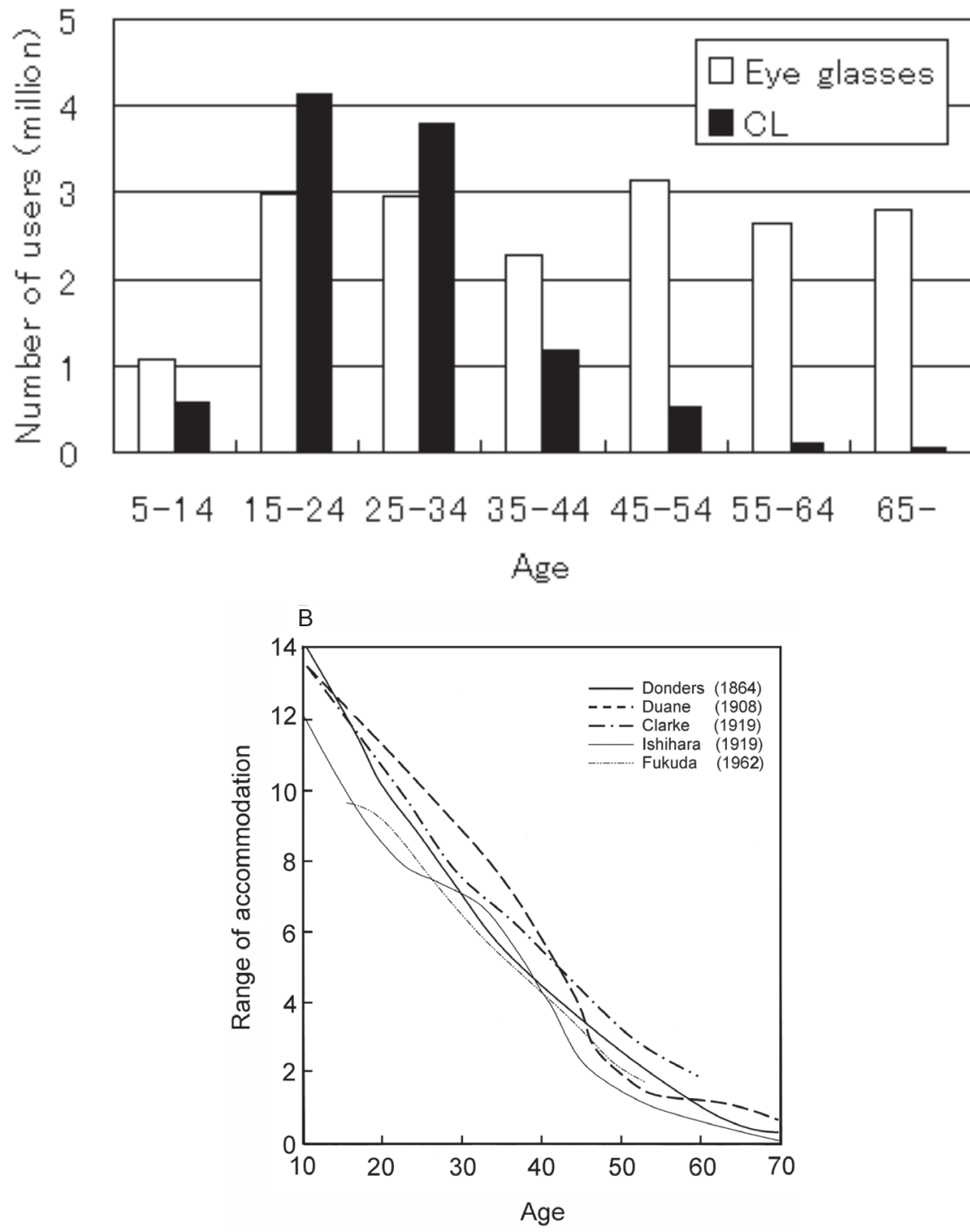

Figure 8 A Number of persons using eye glasses and CL in each age in Japan. B Age versus accommodation curve. The number of CL wearers decreases at the age of 35 years or more, and shows a further decrease at the age of 45 years or more in Japanese. It decreases markedly when presbyopia becomes a problem, and the pattern of decrease is very similar to the age versus accommodation curve. 
cal SCL when either a SofLens ${ }^{\circledR}$ Multi-Focal or Acuvue ${ }^{\circledR}$ Bifocal was prescribed (Edmunds 2003; Situ et al 2003). In particular, Edwards and Orsborn (2003) reported that the SofLens ${ }^{\circledR}$ Multi-Focal demonstrated superiority to all other lenses in terms of vision at any distance and was preferred by patients and practitioners in 12 countries.

There are also lenses designed to provide modified monovision such as the Frequency ${ }^{\circledR} 55$ Multifocal equipped with a D-lens for distant vision in the center for the dominant eye and an N-lens with near vision in the center for the nondominant eye (Iravani 2002), as well as the UltraVue ${ }^{\mathrm{TM}}$ 2000 Toric Multifocal equipped with a D-lens (distant vision) and an N-lens (near vision) in the center, and a toric design for the posterior part of the lens (Choate 2002). As for the technique of selecting a lens with modified monovision, Quinn (2002) has proposed adding power for distant and near vision when necessary with small additions for the dominant eye (distant vision) and sufficient extra power for the nondominant eye (near vision). Walker (2002) has reported success with prescribing a toric CL for distant vision and bifocal CL for the other eye in patients with astigmatism.

In recent years, bifocal SCL made of silicone hydrogel have also become available. This type may be effective in persons with mild dry eyes. According to the results of a US clinical study of PureVision Multifocal TM (Bausch Lomb 2007), it could be worn continuously for 1 year by $75 \%$ of subjects, and symptoms like dryness and a foreign body sensation improved over time. Thus, the contact lens is improving and evolving.

Increased use of LASIK (laser in situ keratomileusis) was forecast to make CL unnecessary at some point, but it has yet to be reached. This may be partly because standard LASIK cannot correct for presbyopia. Although LASIK with correction of presbyopia and bifocal intraocular lenses have become have been available recently, irreversibility is a disadvantage of both procedures. Correction of high aberration also appeared in the latest method of wavefront-guided LASIK, but it may be better to leave this uncorrected and rely on pseudo-accommodation. On the other hand, use of bifocal CL allows changing the prescription as often as needed, which is a great advantage, although patience is required to achieve success.

In the present study, the mean age of the patients who wore bifocal CL for at least 3 years was 50 years, suggesting that mild to moderate presbyopia is a good indication for bifocal CL. However, it is also important to select eyes that are indicated for bifocal CL, because the incidence of problems like glare and poor vision is by no means low.
Before prescribing bifocal CL, their limitations should be understood, including limited addition for near vision, as well as the effects of aging and eye diseases such as astigmatism, dry eyes, cataract, glaucoma, and age-related macular degeneration, etc. Bifocal CL also appears to be unsuitable for anxious people or perfectionists even when they have none of these diseases. Prescription can be considered successful if wearers have adequate distant and near vision, although neither will be perfect.

\section{Acknowledgment}

Presented in part at the meeting of XXX international Congress of Ophthalmology, February 19-24, 2006, Sao Paulo, Brazil. The authors declare no conflicts of interest.

\section{References}

Back AP, Holden BA, Hine NA. 1989. Correction of presbyopia with contact lenses comparative success rates with three systems. Optom Vis Sci, 66:518-25.

Clarke E. 1918. The errors of accommodation and refraction of the eye and their treatment. London: Bailliere, Tindall and Cox, pp. 142-51.

Choate WL. 2002. Astigmatism and presbyopia: contact lens double jeopardy? CL Spectrum, 17:21.

DeCarle J. 1989. Bifocal and multifocal contact lens, In: Stone J, Anthony PJ (eds). Contact lenses. 3rd ed, London: Butterworths, pp. 595-624.

Donders FC. 1864. On the anomalies of accommodation and refraction of the eyes. London: The New Sydenham Society, pp. 87-93.

Duane A, 1908. An attempt to determine the normal range of accommodation at various ages, being a revision of Donder's experiments. Trans Am Ophthalmol Soc, 11(Pt 3):634-41.

Edmunds FR. 2003. A clinical assessment of presbyopic soft contact lens designs. Rev Optom, 140(4 part 2 of 2):2-15.

Edwards K, Orsborn G. 2003. Correcting presbyopes with soft contact lenses-can we do better? Optician, 226:22-6.

Freeman M, Stone J. 1987. A new diffractive bifocal contact lens. Transactions BCLA Conference, 15-22.

Fukuda M, Hamada Y, Maruo T. 1962. Accommodation-age relation of Japanese. J Jpn Ophthalmol Soc, 66:181-8.

Gohrmlet NR. 1989. The hydron ECHELON bifocal contact lens. ICLC $16: 315$.

Hamano T. 2002. Practicality of prescribing a bifocal hard contact lens. J Jpn CL Soc, 44:s16-s20.

Hamano T, Hamano T, Hamano K, et al. 2006. 5-year following-up study of 423 wearers of 2-week replacement bifocal contact lenses. J Jpn CL Soc, 48:2-6.

Harris MG, Kuntz S, Morris C, et al. 2005. Use of presbyopic contact lens corrections in optometric practices. CL spectrum, 20:42-6.

Iravani N. 2002. New multifocal offers best of both worlds. CL Spectrum, (Supplement: 0902 cooper) 17:2-5.

Ishihara S.1919. Accommodation in Japanese eyes. J Jpn Ophthalmol Soc, 28:203-10.

Moss HI. 1962. Bifocal contact lens - A review. Am J Ophtom Arch., 39:653-68.

Quinn TG. 2002. The monovision vs. multifocal debate. CL Spectrum, $17: 19$.

Sado K. 2000. Bifocal or multifocal contact lenses for presbyopia. J Jpn CL Soc, 42:s3-20.

Situ P, DuToit R, Fonn D, et al. 2003. Successful monovision contact lens wearers refitted with bifocal contact lenses. Eye Contact Lens, 29:181-4. 
Stein HA. 1990. The management of presbyopia with contact lens. CLAOJ. $16: 33-9$.

Succeed Co., Ltd. 2004. Eye glasses white paper Japan 2004-2005. Tokyo, Japan: Succeed Co, Ltd., pp. 10-24.

Takahashi K, Sado K. 2002. A clinical evaluation of various multifocal (bifocal) contact lenses. Juntendo Med J, 48:56-6.

Tanaka H, Sawano T, Oyama H et al. 1995. Evaluation of optical decentered bifocal contact lens. J Jpn CL Soc, 37:158-61.

Tanaka H, Hirai Y, Inagaki Y, et al. 1996. Fitting experience with the bifocal contact lens in which the optical axis coincides with the line of sight (Menifocal Soft 72). J Jpn CL Soc, 38:95-8.
Van Meter WS, Gussler JR, Litteral G. 1990. Clinical evaluation of three bifocal contact lenses. CLAO J, 16:203-7.

Van Meter WS, Hainsworth KM, Duff C et al. 2001. Bifocal contact lenses in presbyopia. Int Ophthalmol Clin, 41:71-90.

Walker JS. 2002. Case study: presbyopia and astigmatism. CL Spectrum, $17: 6-8$.

Bausch Lomb Co. Ltd. 2007. PureVision Multifocal ${ }^{\mathrm{TM}}$ package insert [online]. Accessed November 3, 2007. URL: http://www.bausch.com/ en_US/downloads/ecp/visioncare/general/pv_mult_pkg_insert.pdf. 
